\title{
Brucella Species Infection of Ventricular-peritoneal Shunt: A Rare form of Neurobrucellosis Presenting as an Intra-abdominal Mass
}

\author{
Nawal A Al-Kindi ${ }^{* 1}$, Aisha A Al-Bulushi ${ }^{2}$, Qasim S Al-Hinai ${ }^{3}$, Nuha A Al Tahir ${ }^{2}$, Khalsa Al-Ramadhani ${ }^{4}$, \\ Amal S Al-Maani ${ }^{2,5}$ \\ ${ }^{1}$ Medical Microbiology Department, Khowla Hospital, Oman \\ ${ }^{2}$ Peadiatric Infection Disease Department, the Royal Hospital, Oman \\ ${ }^{3}$ Neurosurgery Department, Khowla Hospital, Oman \\ ${ }^{4}$ Radiology Department, Khowla Hospital, Oman \\ ${ }^{5}$ Directorate general for diseases surveillance and control
}

*Corresponding author: Nawal Al-Kindi; nawalakindi@gmail.com

Received 07 January 2021;

Accepted 24 January 2021;

Published 01 February 2021

\begin{abstract}
A 12-year-old girl with right cerebellar pilocytic astrocytoma and left Ventricular - Peritoneal (VP) shunt presented with prolonged progressive painful abdominal distention, constipation, significant weight loss and intermittent fever. Laboratory investigations including blood and CerebroSpinal Fluid (CSF) tests revealed a VP shunt infection with Brucella abortus + Brucella melitensis. Abdominal Computed Tomography (CT) scans revealed a large abdominopelvic cyst with enhancing wall suspicious for infected pseudocyst. Ovarian tumor markers including AFP, CA-125, CA 19-9 and CEA were negative. Brucella was detected in cyst fluid by Real Time -Polymerase Chain Reaction (RT -PCR). The patient had her infected shunt removed, with establishment of External Ventricular Drainage (EVD) and treatment with antibiotics. A final diagnosis of neurobrucellosis was made and she responded well to 6 months therapy of oral doxycycline, oral rifampicin and oral cotrimoxazole. This case highlights a rare occurrence of neurobrucella in patients with VP shunts and excellent response to source control and antimicrobial therapy.
\end{abstract}

Keywords: neurobrucellosis, brucella, ventriuculo-peritoneal, cerebrospinal-fluid, abdominal-mass.

\section{Introduction}

Brucellosis is a bacterial zoonotic disease of humans and animals worldwide caused by Gram negative bacteria belonging to the genus Brucella which is small, nonencapsulated, nonmotile, facultatively intracellular coccobacilli. Brucellosis is endemic in the Middle East, Mediterranean countries, Asia, Africa and Central and South America ${ }^{[1]}$. In the Middle East, the highest incidence of human brucellosis has been reported in Saudi Arabia, whilst Bahrain has reported no brucellosis cases ${ }^{[2,3]}$. In Oman, human brucellosis is an important zoonotic disease which is now not only restricted to the southern Dhofar governorates due to the presence of infected camels, cattle, goats and sheep, as sporadic cases have occurred in all other Governorates ${ }^{[4,5]}$. A recent study by Alrawahi et al ${ }^{[6]}$ recorded the highest prevalence in camels in Dhofar at $3.7 \%$. The first case of human brucellosis was reported in $1979^{[6]}$ and serological evidence of exposure was found to be $1 \%$ in healthy Dhofar individuals in a study of predominantly children ${ }^{[7]}$.
Brucella infection occurs through the ingestion of contaminated raw milk and other dairy products or by direct contact with contaminated tissue, blood, urine, vaginal discharges, aborted fetuses and placentas of infected animals or inhalation of aerosols ${ }^{[8]}$. A retrospective epidemiological study over a three-year period from the Dhofar governorate revealed that $63 \%$ of cases were due to consumption of raw milk and $83 \%$ of patients had contact with live animals ${ }^{[9]}$.

Brucellosis is a multi-system disease commonly affecting the musculoskeletal system with lesser involvement of the Central Nervous System (CNS). The reported prevalence of neurobrucellosis ranges from 1.7 to $10 \%$ of brucellosis cases worldwide ${ }^{[10,11]}$. Furthermore, clinical manifestations of neurobrucellosis which is potentially a life-threatening disease, are variable ranging from asymptomatic to complicated CNS infection. CNS involvement is usually through the hematogenous route. However, few case reports have shown ascending infection through 
ventricular-peritoneal shunt and vice versa where gastrointestinal manifestation arises ${ }^{[12,13]}$.

\section{Case report}

A 12-year-old child presented with right cerebellar pilocytic astrocytoma in November 2018. She had had a programmable left ventricular - peritoneal (VP) shunt inserted and resection of her pilocytic astrocytoma in November 2018. On March 2019, the child had progressive painful abdominal distention, constipation, significant weight loss for a month and intermittent fever for 2 months. She had no acute neurological manifestation. She denied having chest pain, urinary complaints or back pain. Her dietary history included both pasteurized and unpasteurized animal products such as cheese and milk. She had traveled to Salalah (south of Oman) during the summer of 2018, where she drank unpasteurized milk from camels.

The patient was admitted to the gynecology ward in the Royal Hospital in Muscat on March 2019. During admission she had fever spikes of $38.5^{\circ} \mathrm{C}$. An abdominal examination revealed distended abdomen and generalized tenderness. Laboratory investigation showed a leukocytosis (WBC $15.2 \times 10^{9} / \mathrm{L}$ ) which was predominantly neutrophils and a high C-reactive protein of 397 $\mathrm{mg} / \mathrm{L}$ as shown in Table 1. Figures (1) and (2) show the Computed Tomography (CT) of the chest and abdomen which with $9.6 \times 14.8$ x $20.2 \mathrm{~cm}$ unilocular abdominopelvic cyst with wall enhancement, a moderate right and mild left hydronephrosis and moderate right hydroureter secondary to ureteric obstruction by the cyst. Treatment was initiated with Intravenous (IV) vancomycin (500 $\mathrm{mg}$ twice daily) and IV ceftriaxone (500 mg once daily) for suspected infected collection and a decision for US guided drainage was made.

The pediatric infectious disease team was alerted when an incubated pediatric blood culture bottle (Bact/Alert 3D Microbial Identification System) revealed a Gram-negative coccobacillus which was identified as Brucella species after 5 days. Brucella serology showed a strong positive B. abortus + B.melitensis titer > 1280 which was compatible with the diagnosis of brucellosis. Brucella was detected in the cyst fluid by real time-polymerase chain reaction (RT-PCR) whereas PCR and culture for Mycobactertium tuberculosis were negative. As shown in Table 1, considering the imaging,diagnostic possibility of ovarian mucinous cyst as a less likely differential diagnosis, ovarian tumor markers were assessed including AFP, CA-125, CA 19-9 and CEA and were also negative. Histopathology analysis of the cyst fluid revealed predominantly polymorphonuclear cells along with scattered foamy macrophages.
Brucellosis treatment was started with IV gentamycin (160 $\mathrm{mg}$ once daily for 2 weeks), oral doxycycline (100 mg once daily) and rifampicin (600 $\mathrm{mg}$ once daily). After 2 weeks, the neurosurgery team was alerted and lumber puncture (LP) was performed. Figures $(3,4)$ shows the brain CT and MRI with evidence of obstructive hydrocephalus . A VP shunt revision was advised. Clinically, there were no neurological deficits, apart from the right eye nystagmus. A head CT scan confirmed functioning VP shunt without hydrocephalus as shown in figure (3). However, removal of the VP shunt was done and intra-operational findings suggested an infection at VP shunt site so an external ventricular drain (EVD) was inserted following shunt removal .The CSF fluid analysis listed in Table 1 revealed a positive Brucella serology. A follow up CT head scans showed no ventricular dilation and the EVD was then removed, figure (5).

As her visit to the Brucella endemic area preceded her presentation with cerebellar pilocytic astrocytoma there was concern that the mass could have been part of neurobrucella so review of the tumor tissue was requested. The review of her tumor tissue removed on November 2018 confirmed that histopathology of the right cerebellum of the brain was of pilocytic astrocytoma, WHO grade I with infiltration of the adjacent cerebellar parenchyma. There were no macrophages or inflammatory cells of brucella detected suggesting that this tumor was not related to her later diagnosis with Brucella.

Neurobrucellosis treatment was initiated at least 3 weeks prior VP shunt removal and included a one week of IV ceftriaxone (1g twice daily) and IV ciprofloxacin (400 mg twice daily). EVD was inserted for a while and then clamping challenge was done and was tolerated well by patient. Hence, there was no need for CSF diversion device. This antibiotic treatment may be contributed to patient negative CSF brucella PCR results. The patient was then discharged with the following treatment; oral doxycycline (100 mg once daily), rifampicin (600 mg once daily) and oral cotrimoxazole (140 mg twice daily) for a duration of 6 months. She had regular follow up visits where she was seen in outpatient clinics. Figure (6) shows the pelvis MRI image done as follow up after treatment. The patient was asymptomatic and a persistent decrease in Brucella antibodies titer over the 18-month period as well as negative Brucella cultures were noted. On a follow-up examination, the patient was in good clinical condition and she was finally discharged from the clinic. The child did not have relapse after discontinuing therapy for more than 2 years.

The epidemiological investigation by public health team in the patient residency area revealed two adult Brucella cases during same year which could have been from the farm's cattle. This however was not confirmed in Veterinarian investigation.

Table 1: Summarized laboratory investigation

\begin{tabular}{|c|c|c|c|c|c|c|}
\hline Period Test & $\begin{array}{l}1 \text { week prior } \\
\text { admission }\end{array}$ & $\begin{array}{l}1^{\text {st }} \text { day of } \\
\text { admission }\end{array}$ & $\begin{array}{l}5^{\text {th }} \text { day of } \\
\text { admission }\end{array}$ & $\begin{array}{l}\text { 1-week post } \\
\text { treatment initiation }\end{array}$ & $\begin{array}{l}\text { 1-month post VP } \\
\text { shunt removal }\end{array}$ & $\begin{array}{l}6 \text { weeks to } 6 \\
\text { months post VP } \\
\text { shunt removal }\end{array}$ \\
\hline WBC $\left(4.5-14.510^{9} / \mathrm{L}\right)$ & & 15.2 & 9 & 9.8 & 7 & 6 \\
\hline CRP (0-5 mg/L) & & 397 & 328 & 95 & & \\
\hline \multicolumn{4}{|l|}{ ESR (0-20mm/hr) } & 71 & & \\
\hline \multicolumn{3}{|c|}{ Serology (B.abortus +B.mlitensis) titer } & $>1280$ & \multicolumn{2}{|l|}{1.320} & 1.180 \\
\hline Blood culture & & \multicolumn{2}{|c|}{ Brucella species } & Brucella species & Negative & Negative \\
\hline \multicolumn{6}{|l|}{ Cyst fluids : } & \\
\hline \multicolumn{4}{|l|}{ Morphology } & \multicolumn{2}{|l|}{ Turbid } & \\
\hline \multicolumn{4}{|l|}{ Polymorph cells } & \multicolumn{2}{|l|}{ Scanty } & \\
\hline \multicolumn{4}{|l|}{ Culture } & \multicolumn{2}{|l|}{ Negative } & \\
\hline \multicolumn{4}{|l|}{ RT-PCR } & \multicolumn{2}{|l|}{ Detected } & \\
\hline
\end{tabular}




\begin{tabular}{|l|l|}
\hline Tumor markers : & \\
AFP(0-7)mg/ml & 0.5 \\
CA-125(0-35)u/ml & 110 \\
CA 19-9(<37)iu/L & 7 \\
CEA (0-3)ug/L & 0.8 \\
\hline CSF : & \\
Morphology & Clear \\
Protein $(15-45 \mathrm{mg} / \mathrm{dL})$ & 26.58 \\
Glucose $(3.334 .44 \mathrm{mmol} / \mathrm{L})$ & 2.9 \\
WBC & $8 * 10^{9} / \mathrm{L}$ \\
RBC & $600 * 10^{9} / \mathrm{L}$ \\
Culture & No growth \\
Serology( Brucella ) & Positive \\
RT-PCR (Brucella ) & Negative \\
\hline
\end{tabular}

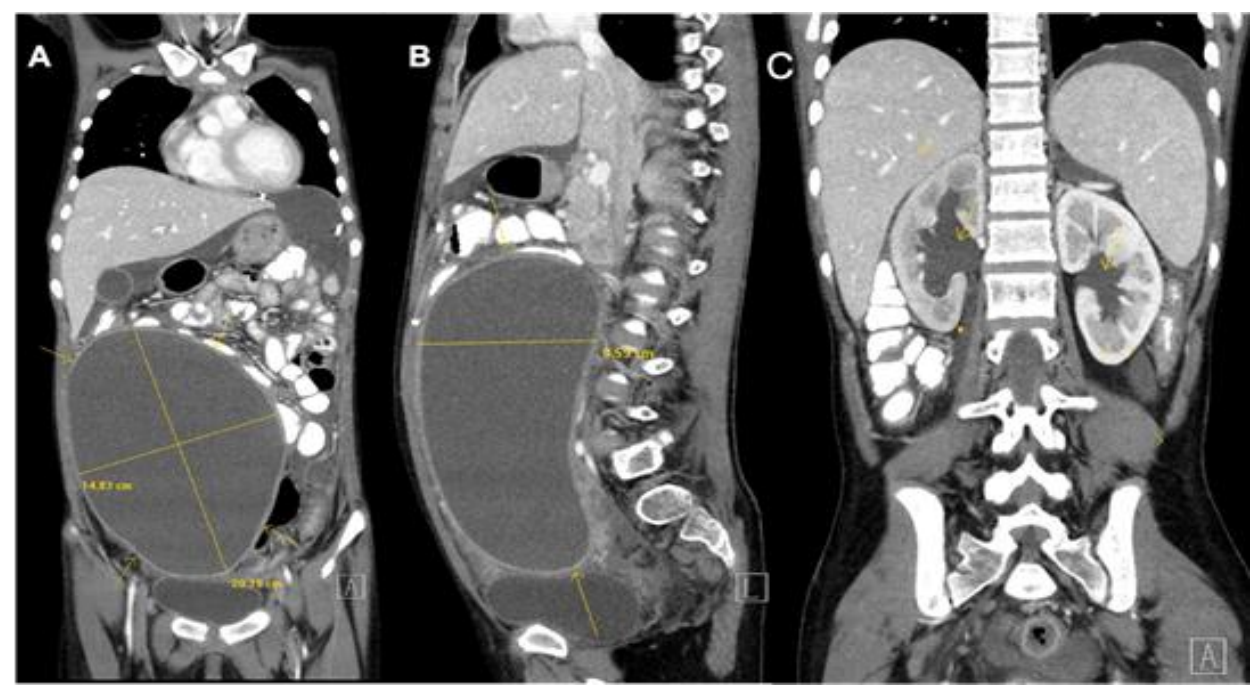

Figure (1) and (2): Coronal (A) and sagittal (B) images of CT chest and abdomen with IV contrast demonstrating 9.6 x $14.8 \times 20.2 \mathrm{~cm}$ unilocular abdominopelvic cyst $\rightarrow$ ) with wall enhancement. Coronal image $(C)$ demonstrates moderate right and mild left hydronephrosis $(\rightarrow)$ and moderate right hydroureter $(*)$ secondary to ureteric obstruction by the cyst.

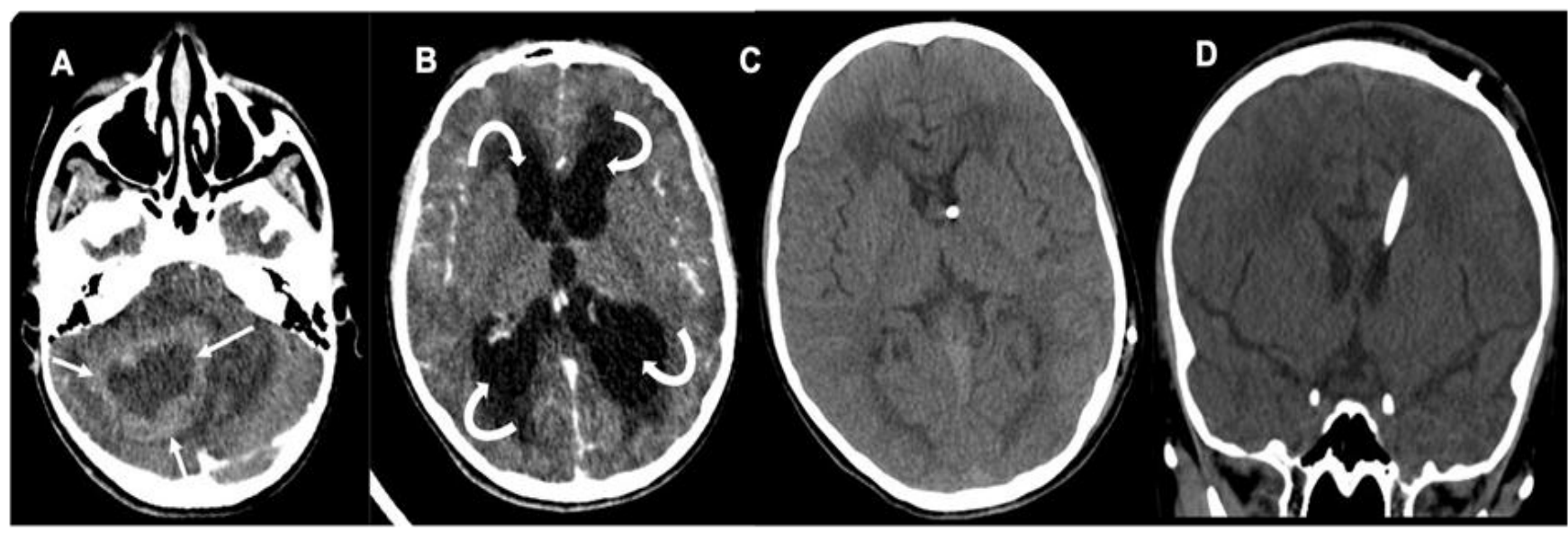

Figure 3: CT scan of the brain with IV contrast (A \& B) demonstrates heterogenous right cerebellar mass $(\rightarrow)$ with cystic and enhancing solid component compressing the fourth ventricle with supratentorial obstructive hydrocephalus (curved arrows). CT scan of the brain without contrast $(C \& D)$ post insertion of VP shunt demonstrates decrease in the size of the ventricles. 


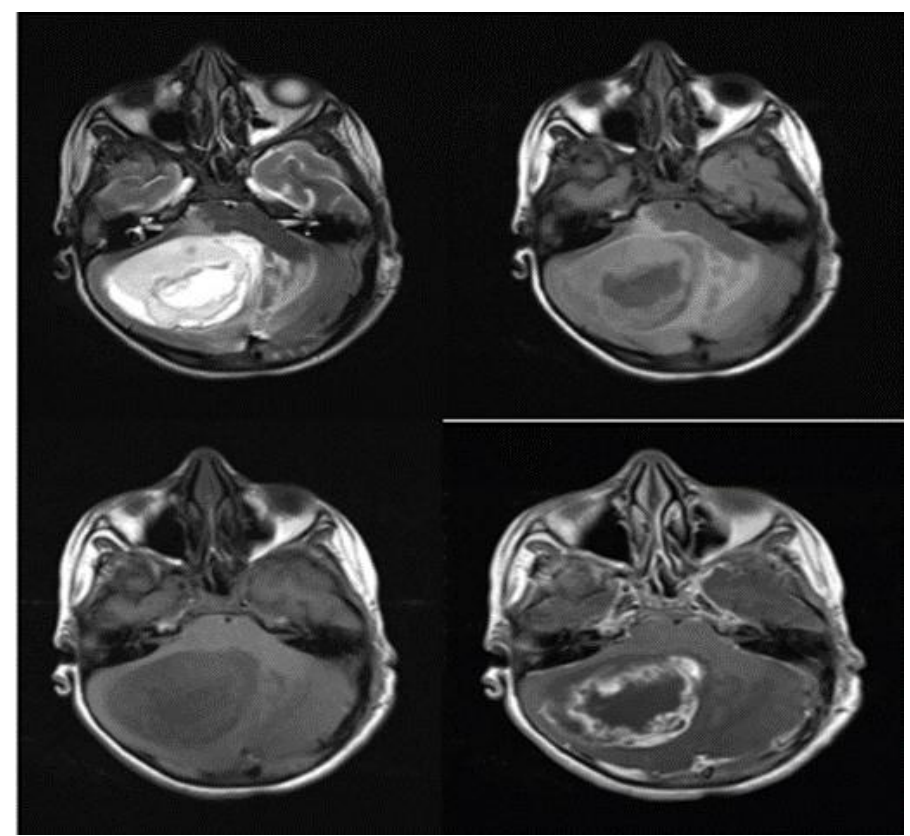

Figure 4: Brain MRI showing the right cerebellar hemispheric mass with cystic and enhancing solid component with surrounding edema compression of the fourth ventricle causing supratentorial hydrocephalus.

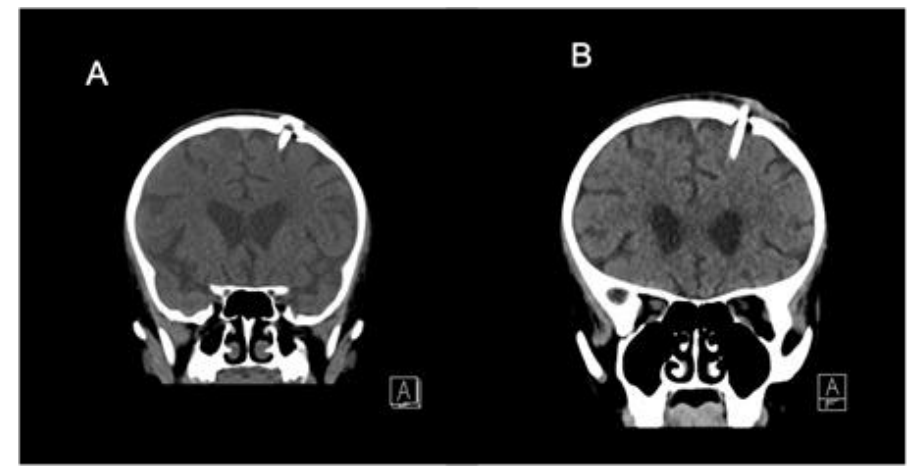

Figure 5: CT scan of the head without contrast with the VP shunt (A) and after removal of the shunt and insertion of EVD (B)

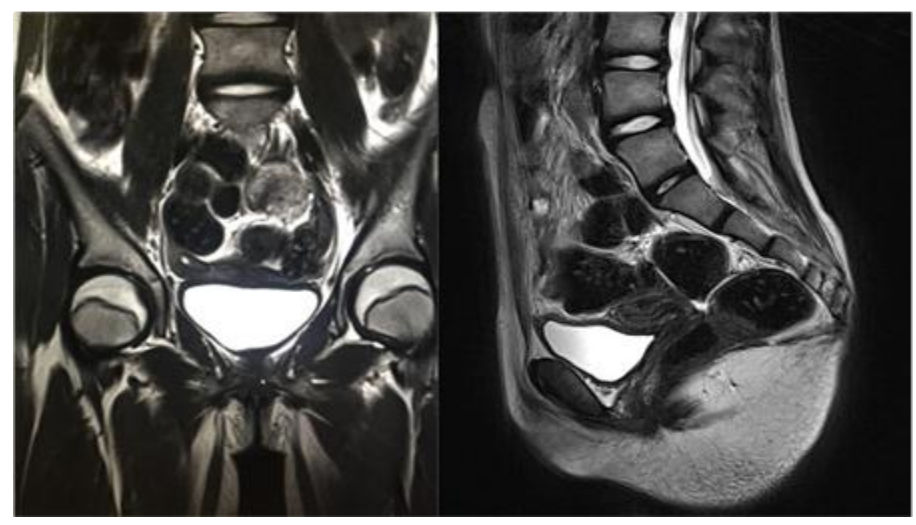

Figure 6: Follow up Pelvis MRI coronal and sagittal T2WI demonstrates complete resolution of the previously seen cyst.

\section{Discussion}

In 2002, the incidence of brucellosis in Oman was reported to be 6.8 per 10000 people ${ }^{[14]}$. Neurobrucellosis occurs in about $7 \%$ of adult cases but is rare at $<2 \%$ of cases in children ${ }^{[15]}$ with mortality rates of between $0-27 \%$ previously reported ${ }^{[16,17]}$. It is known that the clinical spectrum of neurobrucellosis is very heterogeneous and has been described as either central or peripheral forms ${ }^{[11]}$. The central form is commonly acute and results mainly in meningoencephalitis, while the peripheral form may be either acute or chronic in presentation ${ }^{[18]}$. Variable clinical manifestations include meningitis, encephalitis, polyradiculoneuritis, sensorial and motor abnormalities, cranial nerve involvement, epilepsy, brain abscess, subarachnoid hemorrhage as well as coma ${ }^{[19]}$. However, VP shunt infection with Brucella is extremely rare ${ }^{[20]}$. Our patient presented with fever, constipation and abdominal pain which is consistent with previously reported observations [13,21,22]. Nevertheless, in high endemic regions, Brucella should be considered as the causal pathogen. Abdominal cyst formation as seen in our patient occurs very rarely. As the cyst fluid showed the presence of Brucella by PCR, it may be possible that this fluid had the growth of Brucella organisms prior antibiotic treatment which resulted in masking the brucella growth from the cyst fluid ${ }^{[13]}$. 
This case study highlights the importance of testing for Brucella infection in endemic areas as our patient presented with Brucella infection of VP shunt manifesting with fever, abdominal pain and constipation but no associated neurological symptoms. In contrast, Gul et al in $2009^{[23]}$ reporting on a pooled analysis of 187 cases of neurobrucellosis in Turkey found that most presented with neurological symptoms. The diagnosis of neurobrucellosis was confirmed in our patient by the detection of Brucella in blood cultures as well as CSF findings of increased white cells, high protein levels, low glucose and positive Brucella serology. The importance of CSF analysis was highlighted in our case as the management of neurobrucellosis requires appropriate treatment to be started early as well as using antibiotic drug treatments to cross the blood-brain barrier in order to bind their receptors ${ }^{[18]}$. Although a recent case report states that the evaluation of CSF is not mandatory for the diagnosis of neurobrucellosis, the authors suggest that CSF analysis can result in a quicker implementation of appropriate treatment as it is known that delay in proper treatment can result in clinical sequelae including death ${ }^{[24]}$.

Our patient had her infected shunt removed, with establishment of EVD and treatment with antibiotics. The patient responded well to IV ceftriaxone and IV ciprofloxacin followed by 6 months duration of oral doxycycline, oral rifampicin and oral cotrimoxazole on discharge. Proper treatment management as well as prolonged treatment is recommended for treatment of neurobrucellosis for patients to make a complete recovery.

\section{Competing Interest}

The authors declare that they have no competing interests.

\section{Authors' contributions}

All authors have read and agreed to the final manuscript.

\section{Ethics approval and consent to participate}

Not applicable.

\section{List of abbreviations}

VP: Ventricular - Peritoneal

CSF: Cerebro-Spinal Fluid

CT: Computed Tomography

RT-PCR: Real Time -Polymerase Chain Reaction

EVD: External Ventricular Drainage

CNS: Central Nervous System

IV: Intra-Venous

\section{Conflicts of Interest}

The authors declare that there is no conflict of interest regarding the publication of this paper.

\section{Funding}

No funding was required.

\section{Authors' contributions}

Dr Nawal Al-Kindi: writing up the case report, responsible for diagnostic testing, following up case until case clinical, radiological and laboratory management is fully completed.
Dr Aysha Al-Balushi: clinical presentation case report write up. Dr Nuha Al-Taher: clinical management of case in infection disease clinic and general case report review.

Dr Amal Al-Maani: clinical management of infection in infection disease clinic, overall case report review.

Dr Khalsa Al-Ramadhani: Providing radiological images and annotated them .Case report review.

Dr Qasim Al-Hinai: neurosurgical intervention and review of case report.

\section{Acknowledgments}

All institutes were the case was investigated, managed and followed up. These include the Royal Hospital infection disease department, Khowla Hospital medical microbiology department, Khowla hospital neurosurgery department, khowla Hospital radiology department and the Central Public Health Laboratories (CPHL) where the RT-PCR testing was done.

\section{References}

[1] Corbel, JM, Brucellosis: an overview, Emerg Infect Dis, 2: 213-21. 1997.

[2] Refai, M. Incidence and control of brucellosis in the Near East region, Veterinary Microbiology 90 81-110. 2002

[3] Brown CC, Garin-Bastuji B, Ziay G, Hayajneh W, AlFuqaha S, Hemida H, Otify Y, Hailat K, Alkhlouf S, Hailat N. Improving the diagnostic capacities of brucellosis, enhancing the vaccination and control strategies in the Middle East and North Africa. Pakistan Veterinary Journal, 34(2):263-264, 2014

[4] Tahir TE, Remaya RN. Prevalence of brucellosis in Sultanate of Oman with reference to some Middle-East countries. Vet Res. 4(3); 71-76: 2011

[5] Sultanate of Oman, Ministry of Health, Communicable Diseases Manual, Third Edition, 2017. http://www.moh.gov.om/

[6] Alrawahi AH, Robertson I, Hussain MH and Saqib M. A cross-sectional seroepidemiological study of camels (Camelus dromedaries) brucellosis and associated risk factors in the Sultanate of Oman. Open Vet Journal 9 (2): 133-139 (2019)

[7] Scrimgeour E.M., Mehta F.R. \& Suleiman A.J. (1999) Infectious and tropical diseases in Oman: a review. American Journal of Tropical Medicine and Hygiene 61, 920-925.

[8] Şahin M, Unver A, Otlu S. Isolation and biotyping of Brucella melitensis from aborted sheep fetuses in Turkey. Bull Vet Inst Pulawy. 2008;52:59-62

[9] El-Amin EO, George L, Kutty N K, Sharma P P, Choithramani R S, Jhaveri V P, Salil P, Bedairet S M, Brucellosis in children of Dhofar Region, Oman. Saudi Med J Jul;22(7):610-5. 2001

[10] Akdeniz, H, Irmak, H, Anlar, O, Demiröz, AP. Central nervous system brucellosis: presentation, diagnosis and treatment. J Infect. 1998;36(3):297-301

[11] Shakir, RA, Al-Din, ASN, Araj, GF. Clinical categories of neurobrucellosis: a report on 19 cases. Brain. 1987;110 (Pt 1):213-223.

[12] Mehrabian F, Layaee ZA, Ahmadinejad Z. Brucella shunt infection as a rare presentation of neurobrucellosis. Case reports in Infectious Diseases. Article ID 7291483, Volume 2019. 
[13] Al-Otaibi A, Almuneef M, Al Shaalan M. Brucella melitensis infection of ventriculo-peritoneal shunt: A form of neurobrucellosis manifested as gastrointestinal symptoms. J Infect and Public Health, 7; 62-65: 2008

[14] Community Health and Disease Surveillance Newsletter, Ministry of Health, Oman, 2002; 11 (3).

[15] Turel O, Sanli K, Hatipoglu N, Aydogmus C, Hatipoglu $\mathrm{H}$, Siraneci R. Acute meningoencephalitis due to Brucella: case report and review of neurobrucellosis in children. The Turkish Journal of Pediatrics. 52; 426-429: 2010 .

[16] McLean DR, Russell N, Khan MY. Neurobrucellosis: clinical and therapeutic features. Clin Infect Dis 1992 Oct;15(4):582-590

[17] Bodur H, Erbay A, Akinci E, Colpan A, Cevik MA, Balaban N. Neurobrucellosis in an endemic area of brucellosis. Scand J Infect Dis 2003;35(2):94-97.

[18] Gul HC, Erdem H, Gorenek L, Ozdag MF, Kalpakci Y, Avci IY, Besirbellioglu BA, Eyigun CP. Management of neurobrucellosis: An assessment of 11 cases. Internal Med 47; 995-1001, 2008
[19] Samdani PG, Patil S. Neurobrucellosis. Indian Pediatr 40: 565-568, 2003

[20] Alexiou GA, Manolakos I, Prodromou N. Ventriculoperitoneal shunt infection cause by Brucella melitensis. Pediatric Inf Dis J 12; 1120: 2008

[21] Abdinia B, Barzegar M, Maleki M, Behbod H, Oskoui S Association of Brucella meningoencephatis with cerebrospinal fluid shunt infection in a child: a case report. Iranian J of Child Neurology, 7 (1): 35-38: 2013

[22] Al Shaalan M, Memish ZA, Mahmoud SA, Alomari A, Khan MY, Almuneef M, Alalola S. Brucellosis in children: clinical observations in 115 cases. Int J Inf Dis. 6 (3); 182-186: 2002

[23] Gul HC, Erdem H, Bek S. Overview of neurobrucellosis: a pooled analysis of 187 cases. Int J Infect Dis. 13 (6); e339-e343: 2009

[24] Sharma PP, Murali MV, Hamdi T. Neurobrucellosis presenting as pseudotumor cerebri: First report from Oman. Oman Med J. 32 (6); 507-509: 2017 